Artículo

\title{
Inducción de la respuesta de defensa de plantas de cebolla en la interacción con Trichoderma asperellum y Alternaria porri
}

\author{
Valeria Camacho-Luna ${ }^{1}$ \\ Hilda Elizabeth Flores-Moctezuma \\ Mario Rodríguez-Monroy ${ }^{1}$ \\ Roberto Montes-Belmont ${ }^{2 \dagger}$ \\ Gabriela Sepúlveda-Jiménez ${ }^{1 \S}$
}

${ }^{1}$ Departamento de Biotecnología-Centro de Desarrollo de Productos Bióticos-Instituto Politécnico Nacional. Calle CEPROBI núm. 8, Col. San Isidro, Yautepec, Morelos, México. CP. 62731. (vcamachol1100@alumno.ipn.mx; mrmonroy@ipn.mx). ${ }^{2}$ Departamendo de Interacciones Plantas-InsectoCentro de Desarrollo de Productos Bióticos-Instituto Politécnico Nacional. Calle CEPROBI núm. 8, Col. San Isidro, Yautepec, Morelos, México. CP. 62731. (hflores@ipn.mx).

${ }^{\S}$ Autor para correspondencia: gsepulvedaj@ipn.mx.

\section{Resumen}

Alternaria porri causa la enfermedad mancha púrpura en cebolla y los hongos benéficos del género Trichoderma pueden ser usados para su biocontrol, pero es limitado el conocimiento de los mecanismos de la respuesta de defensa en la interacción de plantas de cebolla con Trichoderma y A. porri. En este estudio se evalúo la actividad de las enzimas de defensa: glucanasas, quitinasas, catalasas y peroxidasa en plantas de cebolla en la interacción con el aislado To de Trichoderma asperellum y A. porri. El aislado To de T. asperellum se seleccionó porque destacó por su actividad antagónica contra $A$. porri en comparación con la de otros aislados de $T$. asperellum (TC1 y TC2) y de $T$. harzianum y $T$. atroviridae. Con el método de cultivo dual y papel celofán,el aislado To inhibió el crecimiento micelial de A. porri en 56 y 53\%, respectivamente y mostró actividad micoparasítica. La actividad de las enzimas dependió de la interacción de las plantas de cebolla con el aislado To de T. asperellum y A. porri. La actividad de glucanasas y quitinasas aumentó con el aislado de $T$. asperellum y se reprimió con $A$. porri. La actividad de catalasas se indujo con $A$. porri y la actividad de peroxidasas aumentó con ambos microorganismos. En conclusión, los mecanismos de defensa de plantas de cebolla son regulados por la interacción con T. asperellum y A. porri y ambos microorganismos; aspecto a considerar en el biocontrol de patógenos de cebolla con el aislado To de T. asperellum.

Palabras clave: enzimas antioxidantes, glucanasas, quitinasas.

Recibido: febrero de 2021

Aceptado: abril de 2021 


\section{Introducción}

Las plantas durante su ciclo de vida interactúan constantemente con microorganismos benéficos y patógenos. De acuerdo con sus estilos de vida, los patógenos de las plantas se clasifican en biotróficos y necrotróficos. Los biotróficos obtienen los nutrientes necesarios para su desarrollo de células vivas de la planta hospedera; ejemplos de este tipo de patógenos son los hongos Phytophthora parasitica y Erysiphe spp. (Glazebrook, 2005).

Mientras que los patógenos necrotróficos dañan el tejido vegetal para utilizarlo como una fuente de nutrientes causando necrosis de los tejidos, algunos ejemplos son los hongos Alternaria brassicicola, Botrytis cinerea, Fusarium oxysporum y Sclerotinia sclerotiorum (Glazebrook, 2005; Birkenbihl y Somssich, 2011). La necrosis vegetal es causada por un aumento de las especies reactivas de oxígeno (ERO) que produce la planta en respuesta a la infección por un patógeno necrotrófico. En el tejido vegetal, la capacidad antioxidante aumenta para tolerar la infección. Por el contrario, en las interacciones con biotróficos, con microorganismos mutualistas y endofíticos, las ERO son moléculas señal para inducir una respuesta sistémica en las plantas.

De esta forma, los microrganismos patógenos y benéficos modifican la red de señales de la defensa de las plantas a través de cambios en los niveles de las fitohormonas: ácido salicílico, ácido jamónico y etileno (Glazebrook, 2005; Barna et al., 2012; Vos et al., 2015). Los mecanismos de defensa que se inducen en las plantas involucran el reforzamiento de la pared celular, donde participan las enzimas peroxidasas y polifenol-oxidasas; la degradación de la pared celular del patógeno por las enzimas glucanasas y quitinasas; y de las enzimas antioxidantes para contrarrestar los niveles de las ERO, como son las catalasas, las superoxido dismutasas y las peroxidasas (Sepúlveda-Jiménez et al., 2005; Kumar et al., 2018; Jain y Khurana, 2018).

Entre los microorganismos benéficos, los hongos del género Trichoderma establecen una relación de simbiosis con las raíces de las plantas, lo que conlleva a promover su crecimiento y desarrollo e inducir una respuesta de defensa en las plantas contra el ataque de patógenos e inclusive insectos plaga (Mendoza-Mendoza et al., 2018; Guzmán-Guzmán-Guzmán et al., 2019). Trichoderma presenta actividad antagónica directa contra patógenos a través de mecanismos como la competencia por espacio y nutrientes, el mico-parasitismo y la antibiosis (Harman 2006; GuzmánGuzmán et al., 2019).

Por estos efectos benéficos, Trichoderma es un agente de control biológico de fitopatógenos y los productos a base de este hongo son usados para mejorar la productividad de los cultivos, la calidad nutricional y la resistencia de las plantas al estrés biótico y abiótico y su uso puede ser una estrategia viable y sostenible para reducir el uso de fertilizantes en cultivos hortícolas (Ortega-García et al., 2015; Fiorentino et al., 2018; Sesan et al., 2020).

Dentro de las hortalizas, la cebolla se destaca por su mayor volumen de mercado a nivel mundial (FAOSTAT, 2020) y Alternaria porri es uno hongo patógeno foliar que ocasiona la enfermedad 'mancha púrpura de la cebolla' que afecta hojas, tallos y bulbos. Los síntomas comienzan en las hojas con lesiones de una forma elíptica y de color entre amarillo y café, pero conforme el hongo se desarrolla, las lesiones se tornan a un color rojizo-púrpura. 
Cuando la infección es fuerte, la planta pierde el follaje y en el bulbo se ocasiona una putrefacción semiacuosa, provocando las pérdidas en el cultivo. Para el control de A. porri se usan fungicidas no sistémicos como el mancozeb y el propineb, y sistémicos como el propiconazol y hexaconazol (Priya et al., 2015), pero el patógeno genera resistencia (Chethana et al., 2012; Rodríguez, 2014). Una alternativa de control biológico de $A$. porri es el uso Trichoderma y se conoce que $T$. harzianum muestra capacidad antagónica contra $A$. porri en ensayos in vitro (Imtiaj y Lee, 2008) y que la aplicación foliar de $T$. harzianum antes y después de la inoculación con $A$. porri reduce la incidencia y la severidad de la mancha púrpura en las plantas de cebolla cultivadas en campo (Prakasam y Sharma, 2012) y en invernadero (Abo-Elyousr et al., 2014).

Sin embargo, los estudios que muestren la actividad de las enzimas implicadas en la defensa de las plantas de cebolla en su interacción con A. porri y con T. asperellum son escasos. Bayoumi et al. (2019) reportan que el tratamiento de las plantas de cebolla con una combinación de T. viride y azufre reduce la incidencia de la enfermedad mancha púrpura; y se relaciona con un aumento de la actividad de catalasas y peroxidasas.

Previamente, en nuestro grupo de trabajo, se reportó que el aislado To de T. asperellum obtenido de un cultivo de cebolla promueve el crecimiento de los bulbos de dos variedades de cebolla, con la ventaja de reducir hasta en $50 \%$ el uso de fertilizantes (Ortega-García et al., 2015). El aislado To de T. asperellum también es un agente potencial para el control biológico de Stemphylium vesicarium que causa el tizón foliar en los cultivos de cebolla del estado de Morelos (ZapataSarmiento et al., 2019).

En base a estos resultados, en la presente investigación se evaluó la actividad de las enzimas glucanasas, quitinasas, catalasas y peroxidasas en la interacción de las plantas de cebolla con el aislado To de T. asperellum y A. porri. La actividad antagónica del aislado To de T. asperellum contra A. porri se comparó con otros dos aislados de T. asperellum obtenidos de cultivos de cebolla y de $T$. harzianum y $T$. atroviridae provenientes de árboles de macadamia.

\section{Materiales y métodos}

\section{Origen de los aislados de los hongos}

El aislado To de Trichoderma asperellum de raíces de cebolla se obtuvo por Ortega-García et al. (2015). Mientras que, en este trabajo, se obtuvieron y se identificaron los aislados TC1 y TC2 de T. asperellum de las raíces de plantas de cebolla cultivada en el municipio de Ayala, estado de Morelos, México. En el mismo lugar se obtuvo e identificó el aislado de Alternaria porri de hojas infectadas de plantas de cebolla. Asimismo, los aislados de T. harzianum y T. atroviridae se obtuvieron e identificaron de raíces de árboles de Macadamia sp. cultivados en el municipio de Tlalnepantla, estado de Morelos, México.

\section{Actividad antagónica de los aislados de Trichoderma spp. contra Alternaria porri}

Método de cultivo dual: en cajas Petri con medio de cultivo de papa, dextrosa y agar (PDA, Bioxon) se colocó en un extremo un disco ( $5 \mathrm{~mm}$ de diámetro) de medio de cultivo con micelio de cada aislado de Trichoderma y en el extremo opuesto de la caja Petri, se colocó otro disco del mismo 
tamaño, pero del patógeno A. porri. El control fue cajas Petri inoculadas con sólo el disco de medio de cultivo con micelio del patógeno que se colocó en la misma posición que en el tratamiento de cultivo dual con Trichoderma. Las cajas Petri se incubaron a $28 \pm 2{ }^{\circ} \mathrm{C}$ con un fotoperiodo de $12 \mathrm{~h}$ luz/12 h oscuridad. Para cada aislado de Trichoderma en cultivo dual con A. porri y el control se realizaron seis repeticiones.

Método con papel celofán: en cajas Petri con medio de cultivo Czapek Dox adicionado con Marmite se colocó un disco de papel celofán encima del medio de cultivo y a continuación, en el centro de la caja se colocó un disco ( $0.5 \mathrm{~mm}$ de diámetro) con medio de cultivo y micelio de cada aislado de Trichoderma. Las cajas se incubaron a $28 \pm 2{ }^{\circ} \mathrm{C}$ con un fotoperiodo de $12 \mathrm{~h} \mathrm{luz} / 12 \mathrm{~h}$ oscuridad por tres días. El papel celofán se retiró y se colocó un disco ( $0.5 \mathrm{~mm}$ de diámetro) con medio de cultivo y micelio de $A$. porri en el centro de la caja.

El control fue cajas Petri con medio de cultivo inoculado sólo con el disco ( $0.5 \mathrm{~mm}$ de diámetro) con medio de cultivo y micelio de $A$. porri. Los cultivos se incubaron a $28 \pm 2{ }^{\circ} \mathrm{C}$ con un fotoperiodo de $12 \mathrm{~h}$ luz/12 h oscuridad hasta que en el control se observó que el micelio de $A$. porri cubrió en su totalidad el medio de cultivo de la caja Petri. Para cada aislado de Trichoderma en confrontación con A. porri y el control se realizaron cinco repeticiones.

Los datos de crecimiento micelial del patógeno que se obtuvieron en los tratamientos de cultivo dual y de papel celofán se usaron para calcular el porcentaje de inhibición del crecimiento micelial de acuerdo con El-Katatny et al. (2001). El porcentaje de inhibición del crecimiento micelial del patógeno se define como la diferencia entre el crecimiento del patógeno en presencia de Trichoderma y el crecimiento del patógeno sin Trichoderma multiplicado por 100. Los datos se analizaron mediante el análisis de la varianza (Anova) y la comparación de las medias se realizó mediante la prueba de Tukey y el programa Rstudio (versión 1.2.1335) con el paquete Agricolae.

Ensayo de micoparasitismo: dentro de una caja Petri se colocó una varilla de vidrio en forma de ' $\mathrm{V}$ ' y sobre ella un portaobjetos con dos discos de PDA, uno de los discos de PDA se inoculó con micelio de $A$. porri y el otro disco con micelio del aislado To de $T$. asperellum y ambos discos se cubrieron con un cubreobjetos estéril. Las cajas se incubaron a $28 \pm 2{ }^{\circ} \mathrm{C}$ con un fotoperiodo de 12 h luz/12 h oscuridad.

Cuando se observó contacto entre el micelio de los dos hongos, se realizó la preparación de los frotis (Quiroz-Sarmiento et al., 2008), se observaron con un microscopio óptico y se les tomó fotografías a $20 \mathrm{X}$ y $40 \mathrm{X}$ de aumento.

\section{Inoculación de plantas de cebolla con Trichoderma asperellum}

Las semillas de cebolla de la variedad Crystal White se adquirió con el distribuidor 'Rancho los Molinos', ubicado en Tepoztlán, estado de Morelos, México. Como sustrato para la germinación de las semillas se utilizó una mezcla de Peet Most y Metromix (Professional Growing Mix, Sunshine, Proveedores Hortícolas de Mexico) en una proporción 1:3 (p:p) esterilizado en una autoclave a $15 \mathrm{lb} \mathrm{cm}^{-2}$ por $30 \mathrm{~min}$ y almacenado a $4{ }^{\circ} \mathrm{C}$ hasta su uso. El sustrato se vertió en charolas de germinación de 96 cavidades, en cada cavidad se colocó una semilla y las charolas se incubaron en un invernadero a $29 \pm 3{ }^{\circ} \mathrm{C}$, con riego cada tercer día. 
A los 20 días, las plántulas se trasplantaron en macetas de plástico de 1 L con el mismo sustrato y se mantuvieron bajo las mismas condiciones de invernadero. Una suspensión de 1.7 x $10^{7}$ esporas $\mathrm{ml}^{-1}$ del aislado To de $T$. asperellum se usó como inoculo y las plantas se inocularon en tres momentos: al momento de la siembra, en el trasplante y al tercer mes después de la siembra. Para la inoculación durante la siembra se colocó $1 \mathrm{ml}$ de la suspensión de esporas sobre las semillas y sobre el sustrato alrededor de ellas; en el trasplante, las plántulas se colocaron dentro de la suspensión de esporas por $15 \mathrm{~min}$ y $1 \mathrm{ml}$ de la suspensión de esporas se colocó en el sustrato que rodeó las raíces; y en la tercera inoculación, $1 \mathrm{ml}$ de la suspensión de esporas se colocó alrededor de la raíz. El control fue plantas sin inocular, pero a las semillas y plantas se les aplicó agua destilada estéril. Las plantas se mantuvieron hasta completar tres meses de edad y se usaron para la infección con $A$. porri.

\section{Inoculación de plantas de cebolla con Alternaria porri}

La inoculación se realizó en las hojas, en donde se colocaron tres discos de medio de cultivo con micelio ( $5 \mathrm{~mm}$ de diámetro) de $A$. porri, enseguida se cubrieron con gasa quirúrgica humedecida en agua destilada estéril. Las plantas se mantuvieron por $72 \mathrm{~h}$ en un invernadero con riego por microaspersión a una humedad relativa de $90 \%$, a $29 \pm 4{ }^{\circ} \mathrm{C}$ con luz natural.

Después, las plantas se mantuvieron con una humedad relativa al $50 \%$ por $24 \mathrm{~h}$. Después de cuatro días de la inoculación con A. porri, las plantas de cebolla se trasladaron al laboratorio donde se cortaron las hojas con unas tijeras, se pesaron y se conservaron a $-4{ }^{\circ} \mathrm{C}$ hasta su uso para la determinación de la actividad enzimática. Los tratamientos fueron hojas de plantas: a) sin inocular (control); b) inoculadas con $T$. asperellum al momento de la siembra, en el trasplante y al tercer mes después de la siembra; c) inoculadas con $A$. porri; y d) inoculadas con $T$. asperellum y con $A$. porri, para cada tratamiento se usaron seis hojas.

\section{Evaluación de la actividad enzimática}

Determinación de la actividad de glucanasas y quitinasas: el tejido de las hojas se maceró con nitrógeno líquido hasta lograr un polvo fino y se mezcló con $1 \mathrm{ml}$ de acetato de sodio $50 \mathrm{mM} \mathrm{a} \mathrm{pH}$ 5. La mezcla se centrifugó a $13000 \mathrm{rpm}$ a $4{ }^{\circ} \mathrm{C}$ durante $30 \mathrm{~min}$., el sobrenadante se separó y se usó como el extracto enzimático. El contenido de proteína del extracto se evaluó por el método de Bradford (1976) y la actividad de glucanasas y quitinasas se determinó por el método descrito por El-Katatny et al. (2001). Para el ensayo de la actividad de glucanasas, la mezcla de ensayo contenía $200 \mu \mathrm{l}$ de extracto enzimático y $500 \mu \mathrm{l}$ de laminarina (Sigma-Aldrich) al 5\% disuelta en agua destilada estéril; la mezcla se incubó a $37^{\circ} \mathrm{C}$, por $30 \mathrm{~min}$ a $300 \mathrm{rpm}$. Para la actividad de quitinasas, la mezcla de ensayo $(1 \mathrm{ml})$ se preparó con $500 \mu \mathrm{l}$ del extracto enzimático y $500 \mu \mathrm{l}$ de quitina coloidal (1\%); la mezcla se incubó a $37{ }^{\circ} \mathrm{C}$ durante 7 h a $300 \mathrm{rpm}$.

El producto de la actividad de glucanasas y quitinasas son azucares reductores como la glucosa y la $\mathrm{N}$-acetil glucosamina respectivamente, que se determinaron por una reacción colorimétrica con el ácido dinitrosalicílico (DNS) de acuerdo con Adney y Baker (2008). Asimismo, para calcular la actividad de las enzimas se construyó una curva patrón con glucosa para las glucanasas y con $\mathrm{N}$ acetil glucosamina para las quitinasas. La actividad de glucanasas se expresó en nmol de glucosa $\mathrm{min}^{-1} \mathrm{mg}^{-1}$ de proteína y la actividad de quitinasas en nmol de $\mathrm{N}$-acetil glucosamina $\mathrm{min}^{-1} \mathrm{mg}^{-1}$ de proteína. 
Determinación de la actividad de catalasas: la actividad de catalasas se evaluó de acuerdo con la metodología de Beers y Sizer (1952). Las hojas se maceraron con nitrógeno líquido y se homogeneizó con $2 \mathrm{ml}$ de buffer de fosfatos de sodio $100 \mathrm{mM}$ a $\mathrm{pH} 7$, adicionado con ácido etilendiaminotetraacético $1 \mathrm{mM}$, fluoruro de fenilmetilsulfonilo $1 \mathrm{mM}$ y diclorodifenil tricloroetano $1 \mathrm{mM}$. El extracto se mezcló con $5 \mathrm{mg}$ de polivinilpolipirrolidona: carbón activado 1:1 (p:p), se centrifugó a $13000 \mathrm{rpm}$ a $4{ }^{\circ} \mathrm{C}$, por $30 \mathrm{~min}$ y el sobrenadante se recuperó y se usó como extracto enzimático. La actividad de catalasa se midió por el decremento de la absorbancia a $240 \mathrm{~nm}$ del $\mathrm{H}_{2} \mathrm{O}_{2}$ usado como sustrato.

La actividad de catalasas se calculó considerando el coeficiente de extinción del $\mathrm{H}_{2} \mathrm{O}_{2}(0.04 \mathrm{mM}$ $\mathrm{cm}^{-1}$ ) y se expresó en $\mu$ moles $\mathrm{H}_{2} \mathrm{O}_{2} \mathrm{~min}^{-1} \mathrm{mg}^{-1}$ proteína. Determinación de la actividad de peroxidasas: la actividad se evaluó con la metodología de Stasolla y Yeung (2007). La mezcla de ensayo contenía $100 \mu \mathrm{l}$ del extracto de proteína y $800 \mu \mathrm{l}$ de buffer de acetato de sodio $50 \mathrm{mM}$ (pH $5.2), \mathrm{H}_{2} \mathrm{O}_{2}(0.3 \%)$ y guayacol $1 \mathrm{M}$.

La oxidación del guayacol en presencia de $\mathrm{H}_{2} \mathrm{O}_{2}$ se midió a una absorbancia de $470 \mathrm{~nm}$ en un espectrofotómetro. La actividad de la enzima se expresó como $\mu$ moles de tetraguayacol $\min ^{-1}$ $\mathrm{mg}^{-1}$ de proteína. Los $\mu$ moles de tetraguayacol se calcularon considerando el coeficiente de extinción del tetraguayacol $\left(26.6 \mathrm{mM} \mathrm{cm}^{-1}\right)$.

Los datos de la actividad de quitinasas, glucanasas y catalasas se analizaron mediante el análisis de la varianza (Anova) y la comparación de las medias se realizó mediante la prueba de Tukey. Los datos de la actividad de peroxidasas se analizaron mediante la prueba de Kruskal Wallis y la prueba de Dunn $(p<0.05)$ debido a que los datos no presentaron una distribución normal ni homogeneidad de varianzas. Los análisis estadísticos se realizaron con el programa de Rstudio (versión 1.2.1335) con el paquete Agricolae.

\section{Resultados y discusión}

\section{Actividad antagónica de los aislados de Trichoderma spp. contra Alternaria porri}

Por el método de cultivo dual, se encontró diferencia significativa en la inhibición del crecimiento de $A$. porri que presentan los tres aislados de $T$. asperellum (TC1, TC2 y To) y el aislado de $T$. harzianum en comparación con $T$. atroviridae. Los tres aislados de $T$. asperellum y de $T$. harzuianum inhibieron más de $40 \%$ el crecimiento micelial de $A$. porri. Mientras que, el aislado de T. atroviridae inhibió en $20 \%$ el crecimiento micelial del patógeno. El aislado To de T. asperellum destacó por su mayor capacidad para inhibir el crecimiento del patógeno (56\%) (Figura 1).

En forma similar, diversos estudios muestran que Trichoderma tiene actividad antagónica contra A. porri y que hay diferencia entre especies e inclusive entre la misma especie de Trichoderma. Prakasam y Sharma (2012) muestran que los aislados de $T$. viride y $T$. harzianum obtenidos de cultivos de plantas inhiben el crecimiento radial de A. porri de 18.9 a $55.7 \%$ y de 19.7 a $61.5 \%$, respectivamente.

La actividad antagónica de Trichoderma se debe a diversos mecanismos, como es su alta capacidad de competencia por nutrientes en comparación a la que presentan otros hongos, a la producción de compuestos llamados sideroforos que atrapan el hierro y detienen el crecimiento de otros hongos y por su capacidad de generar ATP para su crecimiento a partir de diferentes fuentes de carbono (Gajera et al., 2013; Contreras-Cornejo et al., 2016). 


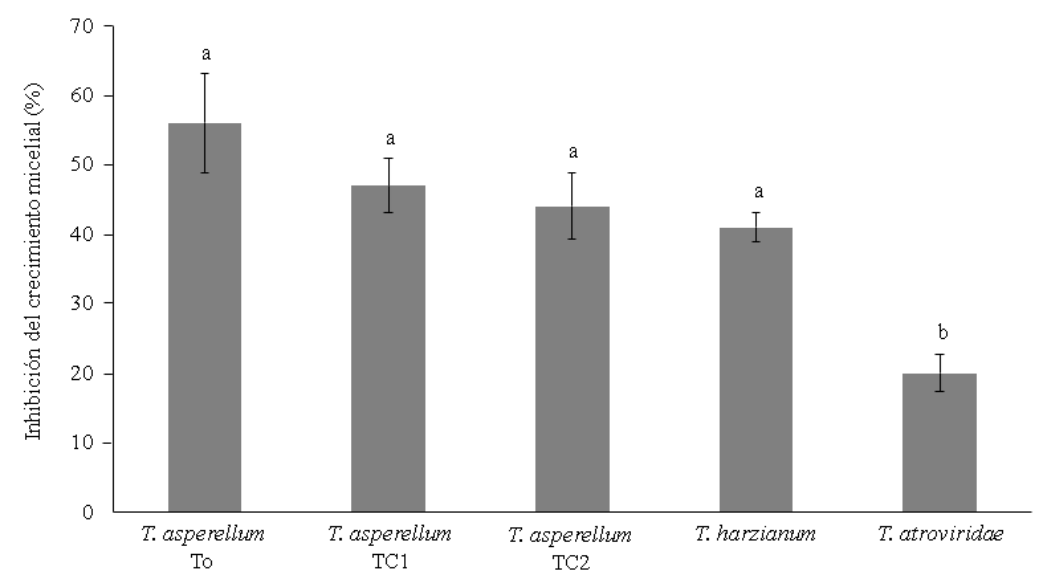

Figura 1. Inhibición del crecimiento micelial de Alternaria porri con diferentes aislados de Trichoderma por el método de cultivo dual. Los valores corresponden a la media y desviación estándar $(n=6)$. Letras diferentes indican diferencias significativas entre los tratamientos de acuerdo con Tukey's HSD test $(p<0.05)$.

Por el método con papel celofán se encontró que hay diferencias significativas en la actividad antagónica de los aislados, pero el aislado To de T. asperellum destacó nuevamente porque inhibió el crecimiento micelial de A. porri en 53\%, mientras que los otros aislados de Trichoderma inhibieron menos de $26 \%$ el crecimiento del hongo fitopatógeno (Figura 2). La variabilidad de la capacidad antagónica de los aislados de Trichoderma que se observó en nuestro estudio puede deberse a que producen diferentes compuestos antimicrobianos que inhiben el crecimiento micelial de A. porri.

Los compuestos con actividad antibiótica pueden ser alquil pironas, isonitrilos, policetidos, peptaiboles, dicetopiperazinas, sesquiterpenos y esteroides (Contreras-Cornejo et al., 2016; Gajera et al., 2013; Harman, 2006). Además de la especie, la producción de estos compuestos depende de la temperatura, el pH y el sustrato en el que se encuentre el hongo (Benítez et al., 2004).

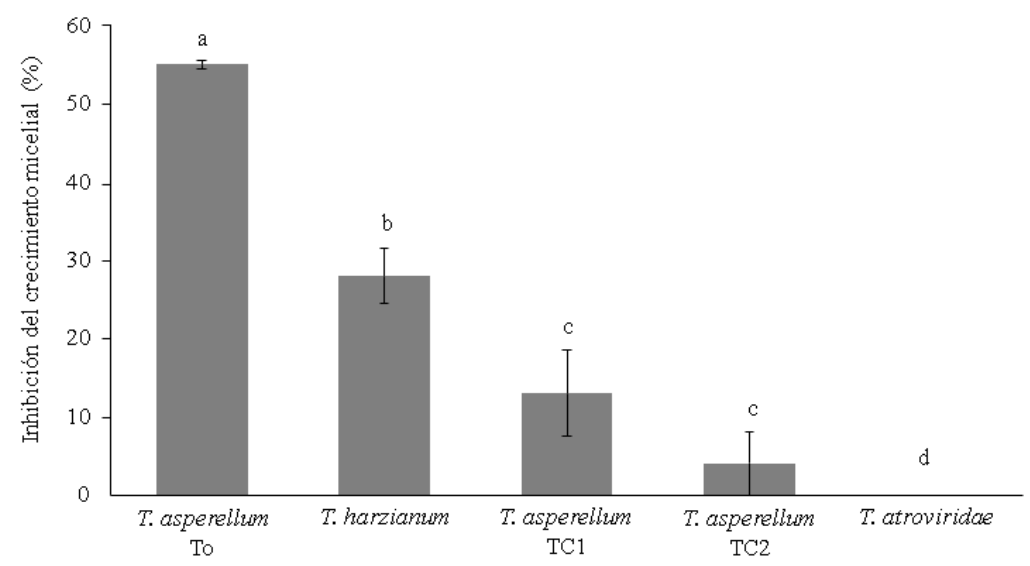

Figura 2. Inhibición del crecimiento micelial de Alternaria porri con diferentes aislados de Trichoderma por el método de papel celofán. Los valores corresponden a la media y desviación estándar $(\mathbf{n}=\mathbf{5})$. Letras diferentes indican diferencias significativas entre los tratamientos de acuerdo con Tukey's HSD test $(p<0.05)$. 
En el proceso de micoparasitismo de T. asperellum se observaron cuatro eventos: el contacto, la invasión, la penetración y la destrucción del micelio de A. porri (Figura 3). Trichoderma tiene potencial para atacar y destruir por micoparasitismo a hongos patógenos de importancia agrícola como Alternaria alternata, Botrytis cinerea, Rhizoctonia solani, Sclerotinia sclerotium, Phytium y Fusarium spp. (Harman et al., 2004; Druzhinina et al., 2011).

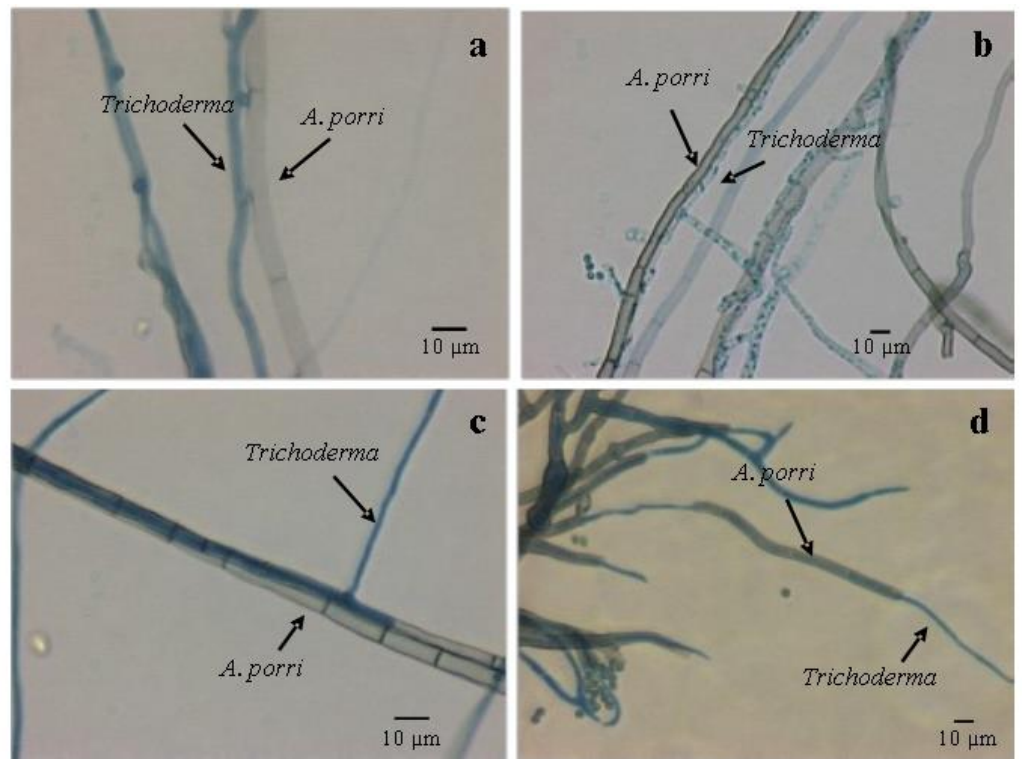

Figura 3. Proceso de micoparasitismo del aislado To de Trichoderma asperellum sobre Alternaria porri. a) Contacto; b) invasión; c) penetración; y d) destrucción.

Los eventos que se observaron en el micoparasitismo de $T$. asperellum sobre $A$. porri se han descrito en otras interacciones de Trichoderma con hongos patógenos y se conoce que es un proceso complejo que involucra la síntesis y reconocimiento de moléculas, la formación de estructuras infectivas y la producción de enzimas y metabolitos antibióticos que dan como resultado la muerte del patógeno (Contreras-Cornejo et al., 2016; Guzmán-Guzmán et al., 2019).

En los primeros momentos de la interacción hay un crecimiento quimiotrófico de las hifas de Trichoderma hacia el patógeno, donde pueden participar señales como las oligoquitinas (Zeilinger y Omann, 2007), cuando las hifas de Trichoderma y del patógeno tienen contacto forman estructuras tipo gancho para unirse al patógeno; en la penetración y la destrucción del micelio del patógeno, Trichoderma produce glucanasas y quitinasas para degradar la pared celular del patógeno (Almeida et al., 2007). Por su actividad antagónica, el aislado de T. asperellum To se seleccionó para estudiar la interacción de T. asperellum y de A. porri con las plantas de cebolla.

\section{Actividad enzimática en plantas de cebolla tratadas con $T$. asperellum y A. porri}

En las plantas inoculadas con T. asperellum se encontraron diferencias significativas en la actividad de las quitinasas y glucanasas en relación con las plantas control. Estas plantas presentaron la mayor actividad de quitinasas y glucanasas seguidas de las plantas no tratadas (control). Mientras que en las plantas infectadas con $A$. porri y las plantas inoculadas con $T$. asperellum e infectadas con $A$. porri se encontró una disminución significativa de la actividad de quitinasas y glucanasas con respecto al control (Figura 4). 

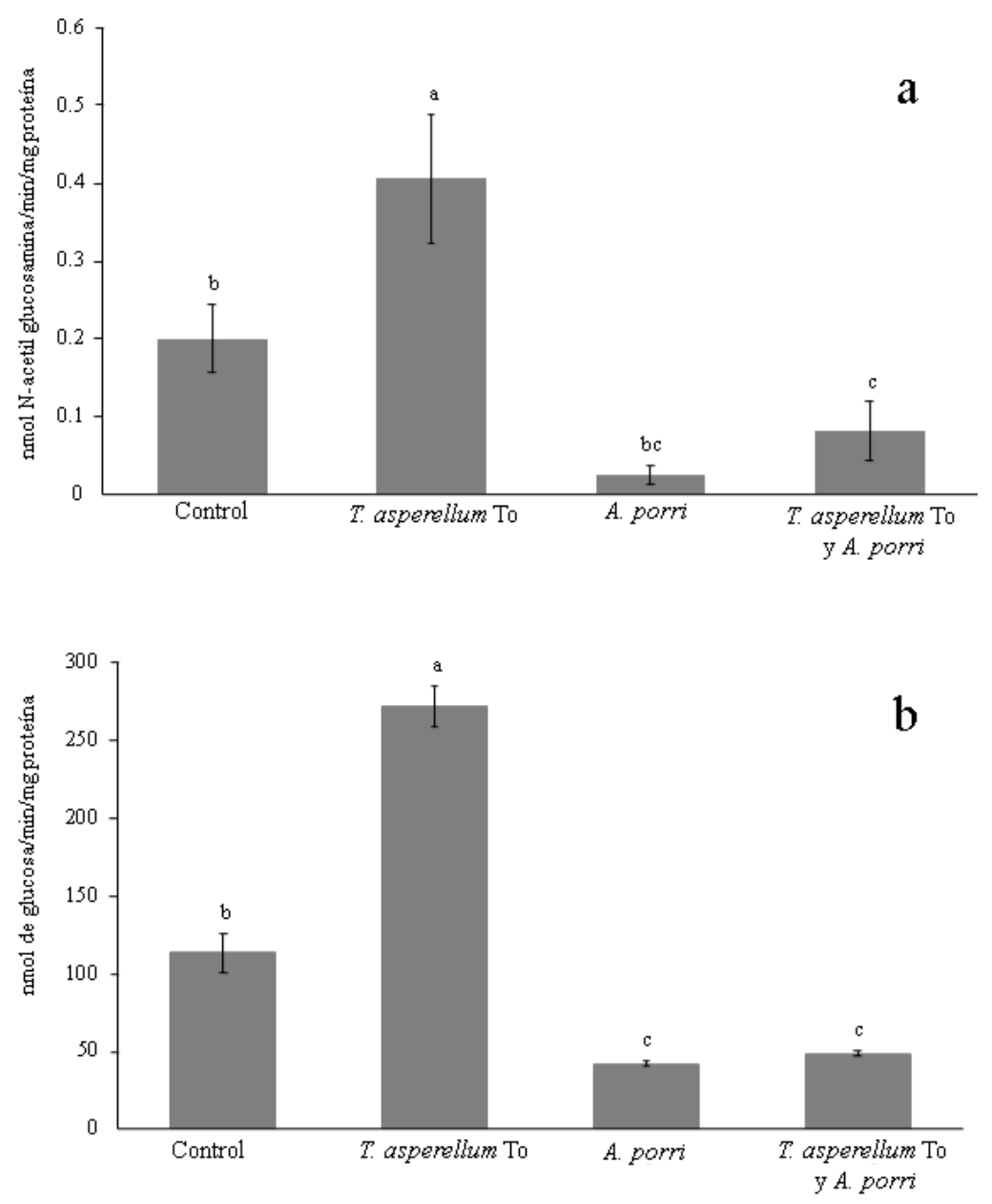

Figura 4) Actividad de: a) quitinasas; y b) glucanasas en hojas de cebolla inoculadas con el aislado To de Trichoderma asperellum, Alternaria porri y con los dos hongos. Los controles son plantas sin inocular. Los valores corresponden a la media y desviación estándar $(n=4)$. Letras diferentes indican diferencias significativas entre los tratamientos con Tukey's HSD test $(p<0.05)$.

Estos resultados indican que la inoculación previa de T. asperellum en las plantas de cebolla induce la actividad de las enzimas de defensa glucanasas y quitinasas para la degradación de la pared celular de hongos patógenos. En forma similar, en otros estudios se reporta que una de las respuestas de defensa en las plantas que se induce por la inoculación de Trichoderma, es la actividad de las enzimas glucanasa y quitinasas (Yedidia et al., 2000; Harman et al., 2004).

Asimismo, se demostró que el aislado TC74 de T. asperellum obtenido de cultivos de Capsicum annuиm (chile) cuando se inocula en plantas de tres variedades de cebolla induce un aumento de la actividad basal de glucanasas, quitinasas y peroxidasas en todos los órganos y esta actividad, se relacionó con una reducción de la severidad de la enfermedad causada por Sclerotium rolfsii (Guzmán-Valle et al., 2014). Sin embargo, la infección con A. porri contrarresta el efecto de Trichoderma en la inducción de la actividad de las enzimas glucanasas y quitinasas, además de causar una reducción de la actividad basal de las dos enzimas que se encontró en las plantas control, lo cual sugiere que $A$. porri cuenta con mecanismos para inhibir la respuesta de defensa basal y la inducida por T. asperellum en las plantas de cebolla. 
Los hongos necrotróficos del mismo género como A. brassicicola y A. alternata producen toxinas específicas que causan la muerte celular del hospedero e inhiben la respuesta de defensa de las plantas (Tsuge et al., 2011; Cho, 2015; Pandey et al., 2016). A. porri también produce toxinas y se ha caracterizado la estructura química, por ejemplo, la entoxina, el silvaticol y el porritoxinol (Suemitsu et al., 1993; 1988), las toxinas inhiben la elongación de raíces de plántulas y la porritoxina de ácido sulfónico presenta una estructura química que se correlaciona con su actividad fito-tóxica (Horiuchi et al., 2003). Sin embargo, no hay estudios de la participación de las toxinas en la patogénesis de A. porri ni en la inhibición de la respuesta de defensa de las plantas; por lo que podría ser un tema de estudios futuros.

La actividad de catalasas mostró diferencias significativas en los distintos tratamientos (Cuadro 1). Las plantas inoculadas con $A$. porri presentaron la actividad de catalasas más alta, seguida de las plantas control, las inoculadas con T. asperellum y las inoculadas con T. asperellum y con A. porri.

Cuadro 1. Actividad de catalasas y peroxidasas en hojas de cebolla inoculadas con el aislado To de Trichoderma asperellum, Alternaria porri y con los dos microorganismos.

\begin{tabular}{lcc}
\hline Tratamiento & $\begin{array}{c}\text { Actividad de catalasas }(\mu \mathrm{mol} \text { de } \\
\mathrm{H}_{2} \mathrm{O}_{2} \mathrm{~min}^{-1} \mathrm{mg}^{-1} \text { de proteína) }\end{array}$ & $\begin{array}{c}\text { Actividad de peroxidasas (nmol de } \\
\text { tetraguayacol } \mathrm{min}^{-1} \mathrm{mg}^{-1} \text { de proteína) }\end{array}$ \\
\hline T. asperellum & $2.14 \pm 0.8 \mathrm{c}$ & $9.93(7.5,12.6) \mathrm{b}$ \\
A. porri & $6.05 \pm 0.5 \mathrm{a}$ & $17.76(14.8,20.9) \mathrm{ab}$ \\
T. asperellum + A. porri & $0.87 \pm 0.2 \mathrm{~d}$ & $35.76(34.1,38.1) \mathrm{a}$ \\
Control & $4.94 \pm 0.2 \mathrm{~b}$ & $7.12(5.5,8.6) \mathrm{b}$ \\
\hline
\end{tabular}

Estos resultados indican que la infección con A. porri induce la actividad de catalasas en las hojas de cebolla; el aumento de la actividad de estas enzimas podría ser un evento ventajoso para $A$. porri, ya que podría evitar la propia muerte del patógeno. Al respecto, se reporta que los hongos necrotróficos del mismo género como son A. alternata, A. brassicae y A. citri producen toxinas específicas (Pandey et al., 2016) que inducen en la planta la peroxidación de lípidos, la acumulación de especies reactivas de oxígeno y la muerte celular.

Sin embargo, el patógeno para evitar su propia muerte por las ERO, coordina las señales para desintoxicar el ambiente oxidante que se genera entre ellos, mediante la activación de enzimas antioxidantes como son las catalasas y el superóxido dismutasas (Chung, 2012).

La actividad de peroxidasas mostró diferencias significativas en los diferentes tratamientos. En las plantas inoculadas con $T$. asperellum y con $A$. porri se encontró la actividad de esta enzimamás alta, seguida de las plantas inoculadas con A. porri, las plantas inoculadas con T. asperellum y el control (Cuadro 1). Los resultados indican que la presencia de ambos microorganismos en las plantas de cebolla induce la actividad de peroxidasas. Estas enzimas están implicadas en los procesos de reforzamiento de la pared celular de las plantas (Jain y Khurana, 2018).

La colonización de las raíces por Trichoderma induce cambios en el proteoma de las plantas y la expresión de las peroxidasas es regulada como parte de los mecanismos de defensa de las plantas (Shoresh y Harman, 2008). Este incremento de la actividad de las peroxidasas por Trichoderma se relaciona con un aumento de la resistencia de las plantas a fitopatógenos (Patel y Saraf, 2017). 
En plantas de cebolla se encontró que el mayor aumento de la actividad de peroxidasas en bulbos y raíces ocurrió cuando las plantas se inocularon con el aislado de $T$. asperellum TC74 y el patógeno Sclerotium rolfsii y se relacionó con una reducción de la severidad de la enfermedad. En la variedad de cebolla no pigmentada hay un mayor aumento de la actividad de peroxidasas que el que se detecta en las variedades de cebollas pigmentadas (Guzmán-Valle et al., 2014).

\section{Conclusiones}

El aislado To de T. asperellum mostró la mayor actividad antagónica contra $A$. porri en comparación a otros aislados de la misma especie y del mismo cultivo de cebolla y de los aislados de $T$. harzianum y $T$. atroviridae. En las plantas de cebolla, se inducen diferentes mecanismos de respuesta de defensa que dependen de su interacción con el aislado To de T. asperellum y A. porri o ambos microorganismos. La inoculación con el aislado To de T. asperellum aumenta la actividad de glucanasas y quitinasas; pero la inoculación con A. porri reprimió la actividad de ambas enzimas. La inoculación con A. porri induce la actividad de catalasas, pero la inoculación con $T$. asperellum y con $A$. porri aumenta la actividad de peroxidasas. Basados en estos resultados, algunos de los aspectos importantes a considerar en un futuro para la aplicación del aislado To de T. asperellum como un agente de control biológico de patógenos de cebolla, podría ser la variedad de cebolla, la nutrición y el tipo de patógeno (biotrófico o necrotrófico).

\section{Agradecimientos}

La investigación recibió el apoyo económico de la Secretaría de Investigación y Posgrado del Instituto Politécnico Nacional (SIP-IPN, proyecto SIP 20200701). MR-M y GS-J agradecen a EDIIPN y SIBE-IPN. VCL es becario de CONACYT (México) para realizar estudios de doctorado y recibió el apoyo del programa de Beca de Estímulo Institucional de Formación de Investigadores (BEIFI-IPN).

\section{Literatura citada}

Abo-Elyousr, K. A. M.; Abdel-Hafez, S. I. I. and Abdel-Rahim, I. R. 2014. Isolation of Trichoderma and evaluation of their antagonistic potential against Alternaria porri. J. Phytophatol. 162(9):567-574. https://doi.org/10.1111/jph.12228.

Adney, B. and Baker, J. 2008. Measurement of cellulase activities. Laboratory analytical procedure. NREL/TP-510-42628. National Renewable Energy Laboratory. Golden, Colorado. 1-8 pp.

Almeida, F. B. R.; Cerqueira, F. M.; do Nascimento-Silva, R.; Ulhoa, C. J. and Lima, A. L. 2007. Mycoparasitism studies of Trichoderma harzianum strains against Rhizoctonia solani: evaluation of coiling and hydrolytic enzyme production. Biotechnol Lett. 29(8):1189-1193. https://doi.org/10.1007/s10529-007-9372-z.

Barna, B.; Fodor, J.; Harrach, B. D.; Pogány, M. and Király, Z. 2012. The Janus face of reactive oxygen species in resistance and susceptibility of plants to necrotrophic and biotrophic pathogens. Plant Physiol. Biochem. 59:37-43. https://doi: 10.1016/j.plaphy.2012.01.014.

Beers, R. F. and Sizer, I. W. 1952. A spectrophotometric method for measuring the breakdown of hydrogen peroxide by catalase. J. Biol. Chem. 195:133-140. 
Benítez, T.; Rincón, A. M.; Limón, M. C. and Codón, A. C. 2004. Biocontrol mechanisms of Trichoderma strains. Int. microbiol. 7:249-260. http://scielo.isciii.es/scielo.php? script=sci_ arttext\&pid=S113967092004000400003\&lng=es\&tlng=es.

Birkenbihl, R. P. and Somssich, E. 2011. Transcriptional plant responses critical for resistance towards necrotrophic pathogens. Front Plant Sci. 2(76):1-5. https://doi.org/10.3389/ fpls.2011.00076.

Bradford, M. M. 1976. A rapid and sensitive method for the quantitation of microgram quantities of protein utilizing the principle of protein-dye binding. Anal. Biochem. 7(72):248-54. Doi:10.1006/abio.1976.9999.

Chethana, B. S.; Ganeshan, G.; Rao, A. S. and Cellishree, K. 2012. In vitro evaluation of plant extracts, bioagents and fungicides against Alternaria porri (Ellis) Cif., causing purple blotch disease of onion. Pest Manag. Hortic. Ecosyst. 18:194-198.

Cho, Y. 2015. How the nectrotrophic fungus Alternaria brassicicola kills plants cells remains an enigma. Eukaryot Cell. 14(4):335-344. Doi:10.1128/EC.00226-14.

Chung, K. R. 2012. Stress response and pathogenicity of the necrotrophic fungal pathogen Alternaria alternata. Scientifica. 1-17. https://doi.org/10.6064/2012/635431.

Contreras-Cornejo, H. A.; Macías-Rodríguez, L.; del-Val, E. and Larsen, J. 2016. Ecological functions of Trichoderma spp. and their secondary metabolites in the rhizosphere: interactions with plants. FEMS Microbiol. Ecol. 92(4):1-21. https://doi.org/10.1093/ femsec/fiw036.

Druzhinina, I. S.; Seidl-Seiboth, V.; Herrera-Estrella, A.; Horwitz, B. A.; Kenerley, C. M.; Monte, E.; Mukherjee, P. K.; Zeilinger, S.; Grigoriev, I. V. and Kubicek, C. P. 2011. Trichoderma: the genomics of opportunistic success. Nat. Rev. Microbiol. 9:749-759. https://doi.org/10.1038/nrmicro2637.

El-Katatny, M. H.; Gudelj, M.; Robra, K. H.; Elnaghy, M. A. and Gübitz, G. M. 2001. Characterization of a chitinase and endo- $\beta-1,3$-glucanase from Trichoderma harzianum Rifai T24 involved in control of the phytopathogen Sclerotium rolfsii. Appl. Microbiol. Biotechnol. 56:137-143. https://doi.org/10.1007/s002530100646.

FAOSTAT. 1997. Statistical database. http://www.fao.org/faostat/en/\#search/onion\%20 production.

Fiorentino, N.; Ventorino, V.; Woo, S. L.; Pepe, O.; De Rosa, A.; Giola, L.; Romano, I.; Lombardi, N.; Napolitano, M.; Colla, G. and Rouphael, Y. 2018. Trichoderma-Based biostimulants modulate rhizosphere microbial populations and improve $\mathrm{N}$ uptake efficiency, yield, and nutritional quality of leafy vegetables. Front Plant Sci. 9:1-15. https://doi.org/10.3389/fpls.2018.00743.

Gajera, H.; Domadiya, R.; Patel, S.; Kapopara, M. and Golakiya, B. 2013. Molecular mechanism of Trichoderma as bio-control against phytopathogen system-a review. Curr. Res, Microbiol. Biotechnol. 1(4):133-142.

Glazebrook, J. 2005. Contrasting mechanism of defense against biotrophic and necrotrophic pathogens. Annu. Rev. Phytopathol. 43:205-27. https://doi.org/10.1146/annurev. phyto.43040204.135923.

Guzmán-Guzmán, P.; Porras-Troncoso, M. D.; Olmedo-Monfil, V. and Herrera-Estrella, A. 2019. Trichoderma species versatile plant symbionts. Phytopathology. 109(1):6-16. https://doi.org/10.1094/PHYTO-07-18-0218-RVW. 
Guzmán-Valle, P.; Bravo-Luna, L.; Montes-Belmont, R.; Guigón-López, C. and SepúlvedaJiménez, G. 2014. Induction of resitance to Sclerotium rolfsii in different varieties of onion by inoculation with Trichoderma asperellum. Eur. J. Plant. Pathol. 138:223-229. https://doi.org/10.1007/s10658-013-0336-y.

Harman, G. E.; Petzoldt, R.; Comis, A. and Chen, J. 2004. Interactions between Trichoderma harzianum strain T22 and maize inbred line Mo17 and effects of these interactions on diseases caused by Pythium ultimum and Colletotrichum graminicola. Phytopathology. 94(2):147-153. https://doi.org/ 10.1094/PHYTO.2004.94.2.147.

Harman, G. E. 2006. Overview of mechanisms and uses of Trichoderma spp. Phytopathology. 96(2):190-194. https://doi.org/ 10.1094/PHYTO-96-0190.

Horiuchi, M.; Ohnishi, K.; Iwase, N.; Nakajima, Y.; Tounai, K.; Yamashita, M. and Yamada, Y. 2003. A novel isoindoline, porritoxin sulfonic acid, from Alternaria porri and the structurephytotoxicity correlation of its related compounds. Biosci. Biotechnol. Biochem. 67(7):1580-1583. https://doi.org/ 10.1271/bbb.67.1580.

Imtiaj, A. and Lee, T. S. 2008. Antagonistic effect of three Trichoderma species on the Alternaria porri pathogen of onion blotch. World J. Agric. Res. 4(1):13-17.

Jain, D. and Khurana, J. P. 2018. Role of pathogenesis-related (PR) proteins in plant defense mechanism. In: Singh, A. and Singh, I. (Ed.). Molecular aspects of plant-pathogen interaction. Springer, Singapore. 265-281 pp. https://doi.org/10.1007/978-981-107371-7_12.

Kumar, M.; Brar, A.; Yadav, M.; Chawade, A.; Vivekanand, V. and Pareek, N. 2018. ChitinasesPotential candidates for enhances plant resistance towards fungal pathogens. Agriculture. 8(7):88. https://doi.org/10.3390/agriculture8070088.

Mendoza-Mendoza, A.; Zaid, R.; Lawry, R.; Hermosa, R.; Monte, E.; Horwitz, B. A. and Mukherjee, P. K. 2018. Molecular dialogues between Trichoderma and roots: role of the fungal secretome. Fungal Biol. Rev. 32(2):62-85. https://doi.org/10.1016/j.fbr.2017.12.001.

Ortega-García, J. G.; Montes-Belmont, R.; Rodríguez-Monroy, M.; Ramírez-Trujillo, J. A.; Suárez-Rodríguez and Sepúlveda-Jiménez G. 2015. Effect of Trichoderma asperellum applications and mineral fertilization on growth promotion and the contect of phenolic compounds and flavoboids in onions. Sci. Hortic. 195(12):8-16. https://doi.org/10.1016/ j.scienta.2015.08.027.

Pandey, D.; Rajendran, S. R. C. K.; Gaur, M.; Sajeesh, P. K. and Kumar, A. 2016. Plant defense signaling and responses against necrotrophic fungal pathogens. J. Plant Growth Regul. 35:1159-1174. https://doi.org/10.1007/s00344-016-9600-7.

Patel, S. and Saraf, M. 2017. Biocontrol efficacy of Trichoderma asperellum MSST against tomato wilting by Fusarium oxysporum f. sp. Lycopersici. arch phytopathology plant protect. 50:228-238. https://doi.org/10.1080/03235408.2017.1287236.

Prakasam, V. and Sharma, P. 2012. Trichoderma harzianum (TH-3) a potential strain to manage the purple blotch of onion (Allium cepa L.) caused by Alternaria porri under North Indian plains. J. Agric. Sci. 4:266-272. https://doi.org/10.5539/jas.v4n10p266.

Priya, R. U.; Sataraddi, A. and Darshan, S. 2015. Efficacy of non-systemic and systemic fungicides against purple blotch of onion (Allium cepa L.) caused by Alternaria porri (Ellis) Cif. Int. Recent Scientific Res. 6(9):6519-6521.

Quiroz-Sarmiento, V. F.; Ferrera-Cerrato, R.; Alarcón, A. y Lara, M. E. H. 2008. Antagonismo in vitro de Aspergillus y Trichoderma hacia hongos filamentosos que afectan el cultivo de ajo. Rev. Mex. Mic. 26:27-34. 
Rodríguez, M. R. 2014. INIFAP. Paquetes tecnológicos, Cebolla. http://sites.securemgr.com/ folder1134/index.cffuseaction $=3251103 \&$ pageid $=44$.

Sepúlveda-Jiménez, G.; Trejo-Tapia, G; Trejo-Espino, J. L.; Bermúdez-Torres, K. y RodríguezMonroy, M. 2005. La producción de las especies reactivas de oxígeno en las interacciones de las plantas con patógenos. Rev. Mex. Fitopatol. 26(1):2980-3009.

Sesan, T. E.; Oancea, A. O.; Stefan, L. M.; Manoiu, V. S.; Ghiurea, M.; Raut, I; ConstantinescuAruxendi, D.; Toma, A.; Savin, S.; Bira, A. F.; Pomohaci, C. M. and Oancea, F. 2020. Effects of foliar treatment with a Trichoderma plant bioestimulant consortium on Passiflora caerulea L. yield and quality. Microorganisms. 8(1):1-27. https://doi.org/ 10.3390/microorganisms8010123.

Shoresh, M. and Harman, G. E. 2008. The molecular basis of maize response to Trichoderma harzianum T22 inoculation: a proteomic approach. Plant Physiol. 147(4):2147-2163. https://doi.org/10.1104/pp.108.123810.

Stasolla, C. and Yeung, E. C. 2007. Cellular ascorbic acid regulates the activity of major peroxidases in the apical poles of germinating white spruce (Picea glauca). Plant Physiol. Biochem. 45(3-4):188-198. https://doi.org/ 10.1016/j.plaphy.2007.02.007.

Suemitsu, R.; Horiuchi, K.; Ohnishi, K. and Yanagawase, S. 1988. High-performance liquid chromatographic determination of macrosporin, altersolanol A, alterporriol A, B and C in fermentation of Alternaria porri (Ellis) Ciferri. J Chromatogr. 454:406-410. https://doi.org/10.1016/S0021-9673(00)88639-2.

Suemitsu, R.; Ohnishi, K.; Horiuchi, M.; Morikawa, Y.; Sakaki, Y. and Matsumoto, Y. 1993. Structure of porriolide, a new metabolite from Alternaria porri. Biosci. Biotech. Biochem. 57(2):334-335. https://doi.org/10.1271/bbb.57.334.n.

Tsuge, T.; Harimoto, Y.; Akimitsu, K.; Ohtani, K.; Kodama, M.; Akagi, Y.; Egusa, M.; Yamamoto, M. and Otani, H. 2012. Host-selective toxins produced by the plant pathogenic fungus Alternaria alternata. FEMS Microbiol. Rev. 37(1):44-66. https://doi.org/10.1111/j.15746976.2012.00350.x.Epub.

Vos, I. A.; Moritz, L.; Pieterse, C. M. J. and Van-Wees, S. C. M. 2015. Impact of hormonal crosstalk on plant resistance and fitness under multi-attacker conditions. Front. Plant Sci. 6:1-13. https://doi.org/10.3389/fpls.2015.00639.

Yedidia, I.; Benhamou, N.; Kapulnik, Y. and Chet, I. 2000. Induction and accumulation of PR proteins activity during early stages of root colonization by the mycoparasite Trichoderma harzianum strain T-203. Plant Physiol. Biochem. 38(11):863-873. https://doi.org/10.1016/ S0981-9428(00)01198-0.

Zapata-Sarmiento, D. H.; Palacios-Pala, E. F.; Rodríguez-Hernández, A. A.; Medina-Melchor, D. L.; Rodríguez-Monroy, M. and Sepúlveda-Jiménez, G. 2019. Trichoderma asperellum, a potential biological control agent of Stemphylium vesicarium, on onion (Allium cepa L.). Biol Control. 140:104105. https://doi.org/10.1016/j.biocontrol.2019.104105.

Zeilinger, S. and Omann, M. 2007. Trichoderma biocontrol: signal transduction pathways involved in host sensing and mycoparasitism. Gene Regul. Sys. Bio. 8(1):227-234. https://doi.org/ 10.4137/grsb.s397. 